\title{
Effect of elastic inhomogeneity on the surface displacements in the northeastern Japan: Based on three-dimensional numerical modeling
}

\author{
Kachishige Sato ${ }^{1}$, Naoya Minagawa ${ }^{2 *}$, Mamoru Hyodo ${ }^{3}$, Toshitaka Baba ${ }^{4}$, \\ Takane Hori ${ }^{4}$, and Yoshiyuki Kaneda ${ }^{5}$ \\ ${ }^{1}$ Department of Astronomy and Earth Sciences, Faculty of Education, Tokyo Gakugei University, \\ Nukuikita-machi 4-1-1, Koganei, Tokyo 184-8501, Japan \\ ${ }^{2}$ Graduate School of Education, Tokyo Gakugei University, Nukuikita-machi 4-1-1, Koganei, Tokyo 184-8501, Japan \\ ${ }^{3}$ Earth Simulator Center, Japan Agency for Marine-Earth Science and Technology, \\ Showa-machi 3173-25, Kanazawa-ku, Yokohama 236-0001, Japan \\ ${ }^{4}$ Institute for Research on Earth Evolution, Japan Agency for Marine-Earth Science and Technology, \\ Showa-machi 3173-25, Kanazawa-ku, Yokohama 236-0001, Japan \\ ${ }^{5}$ Department of Oceanfloor Network System Development for Earthquakes and Tsunamis, \\ Japan Agency for Marine-Earth Science and Technology, \\ Natsushima-cho 2-15, Yokosuka 237-0061, Japan
}

(Received September 26, 2006; Revised August 10, 2007; Accepted September 12, 2007; Online published October 19, 2007)

\begin{abstract}
In geodetic inversions such as estimation of coseismic slip and/or afterslip distribution on faults, the displacements on the surface calculated under an assumption of homogeneous elastic half space have been mostly used as the Green's functions (GF's). However, this seems not adequate for better estimations of such slip distribution, because the subsurface structures are more or less inhomogeneous, especially those in and around Japan where the structure must be much complicated. In this study, to examine how much the inhomogeneous subsurface structure affects on the surface displacements, we conduct some 3-D finite element calculations with a grid for the region of $1400 \mathrm{~km}(\mathrm{EW}) \times 1200 \mathrm{~km}(\mathrm{NS}) \times 200 \mathrm{~km}($ depth$)$ including the Tohoku and Hokkaido, northeastern Japan. Assuming homogeneous and inhomogeneous elastic models with various values for the Young's modulus and Poisson's ratio, we calculated the surface displacements due to a dip-slip type dislocation of $1 \mathrm{~m}$ on many cell-like subfaults assumed on the interface between the Pacific and land side plates. Comparing the results, we find a large discrepancy in the surface displacements between the homogeneous and inhomogeneous elastic models and less dependency of the surface displacements on the Poisson's ratio. The discrepancy is found to be more than $20 \%$ and can be as large as $\sim 40 \%$ in some cases. Such a large discrepancy indicates that the surface displacements calculated for inhomogeneous elastic medium with realistic subsurface structure, unlike as in usual cases, should be used as the GF's for better geodetic inversions.
\end{abstract}

Key words: Geodetic inversion, Green's function, surface displacement, inhomogeneous subsurface structure, numerical modeling, finite element method.

\section{Introduction}

In geodetic inversions such as estimation of coseismic slip and/or afterslip distribution on subsurface faults from observed displacements on the ground, the surface displacements calculated under an assumption of homogeneous elastic half space have been mostly used as the Green's functions (GF's). For example, Heki et al. (1997) estimated the distributions of coseismic slip and afterslip associated with the 1994 far off Sanriku (Tohoku, northeastern Japan) earthquake ( $M$ 7.6) from surface displacements observed at some Global Positioning System (GPS) sites in Hokkaido and northern portion of Tohoku. Miyazaki et

*Present address: Tokiwa-shobo Corporation, Chishirodaikita 3-21-1, Wakabaku, Chiba 264-0005, Japan.

Copyright (c) The Society of Geomagnetism and Earth, Planetary and Space Sciences (SGEPSS); The Seismological Society of Japan; The Volcanological Society of Japan; The Geodetic Society of Japan; The Japanese Society for Planetary Sciences; TERRAPUB al. (2004a, 2004b), Miura et al. (2004) and Ozawa et al. (2004) estimated the distribution of coseismic slip or afterslip associated with the 2003 Tokachi-oki (Hokkaido, northeastern Japan) earthquake ( $M$ 8.0) based on displacements at many sites of the dense GPS network GEONET (GPS Earth Observation Network; e.g., Miyazaki et al., 1997) operated by the Geographical Survey Institute (GSI), Japan, in Hokkaido and northern Tohoku. Baba et al. (2006) also inverted the land-based GPS displacement data by GEONET together with vertical displacements at two ocean-bottom pressure gauges operated by the Japan Agency for MarineEarth Science and Technology (JAMSTEC) (e.g., Hirata et $a l ., 2002)$ to estimate the afterslip distribution following the same earthquake. In these studies, most authors used as the GF's the theoretical surface displacements calculated for a uniform and homogeneous elastic half space, although a few authors (e.g., Miyazaki et al., 2004a) used those calculated for a horizontally layered elastic media. 
However, the subsurface structure of any region is neither homogeneous nor simply layered, and such inhomogeneity or complexity in the subsurface structure should affect on the surface displacements to a certain extent. For instance, Wald and Graves (2001) numerically estimated and compared the surface displacements caused by distributed slips on a subsurface fault in the San Fernando basin region in California, U.S.A., between the cases of homogeneous and inhomogeneous elastic materials. They found that there should be a large discrepancy of $\sim 30 \%$ at maximum between these two cases. Using finite element modeling technique, Masterlark (2003) also numerically evaluated the sensitivities of surface displacements, caused by dislocations distributed on the plate interface in a subduction zone off Mexico, to some factors such as subsurface inhomogeneity. His results also showed quite large $(\sim 100 \%$ in some portion of his model space) differences in the surface displacements between homogeneous and inhomogeneous elastic materials. Presently the surface displacement data obtained with GPS are usually employed in geodetic inversions, and they have quite high precision, say, a few $\mathrm{mm}$ (horizontal displacements) to several $\mathrm{mm}$ (vertical displacements). Hence, the GF's for the surface displacements used in the inversions should also be quite precise and accurate ones estimated for a realistic subsurface structure incorporating the material inhomogeneity. Using the GF's estimated for homogeneous elastic half space must be inadequate (e.g., Masterlark et al., 2001; Masterlark, 2003), especially in the case of inversions of geodetic data for the regions in and around Japan which are located near plate subduction zones with quite strong subsurface inhomogeneity.

In order to quantitatively estimate the effect of subsurface inhomogeneity on the surface displacements, we have calculated them caused by dislocations on cell-like subsurface faults by using a 3-D finite element grid for the northeastern portion of Japan which incorporates a realistic subsurface structure. The calculations were performed for various setting of the material properties, such as the Young's modulus and Poisson's ratio, for the upper crust (UC), lower crust (LC), upper mantle (UM) and Pacific plate (PL). In this paper, we discuss quantitatively the effect of elastic inhomogeneity of subsurface structure on the surface displacements based on these calculations.

\section{Models and Method of Calculation}

In this study, in order to quantitatively estimate how much the subsurface inhomogeneity affects on the surface displacements, we calculate the surface displacements with a finite element method by using a 3-D finite element grid for the northeastern Japan as a test case. The finite element method is a very powerful tool for calculation of deformation and/or stresses in heterogeneous materials, and has been successfully applied in various fields of science. The method has also been frequently used in the solid earth science, for example, to calculate the stresses and/or deformation in plate-subduction and/or plate-collision zones (e.g., Sato et al., 1981, 1996; Sato, 1988, 1989; Hashimoto, 1984, 1985; Yoshioka and Hashimoto, 1989a, 1989b; Yoshioka et al., 1989; Yoshioka and Suzuki, 1997; Kawachi and

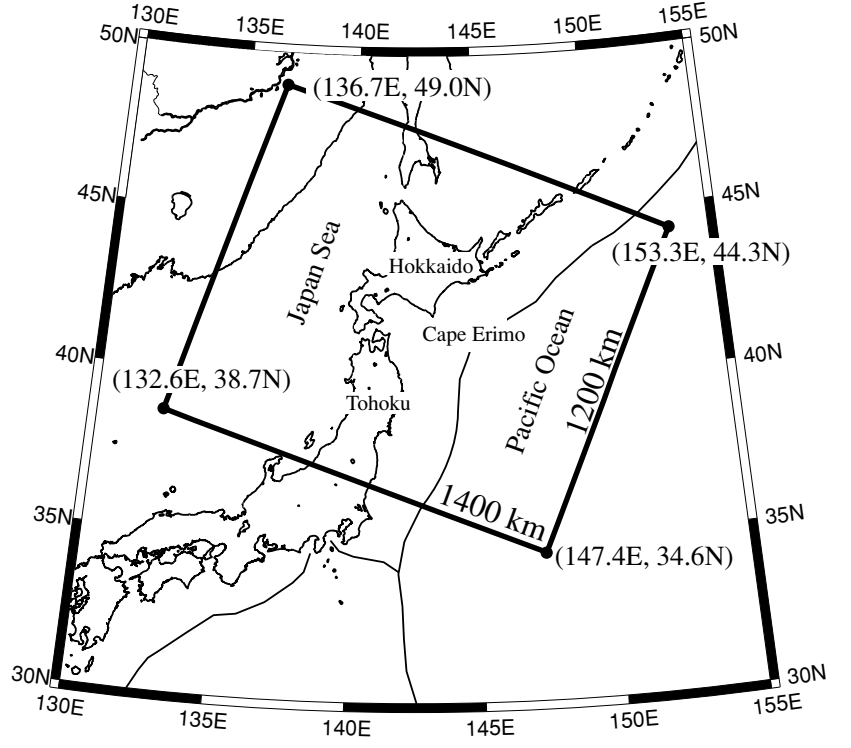

Fig. 1. Map showing the region modeled with a 3-D finite element method in this study. A rectangular region with a dimension of $1400 \mathrm{~km}$ (in $\sim$ EW direction $) \times 1200 \mathrm{~km}$ (in $\sim$ NS direction) indicated by thick lines is modeled.

Miyashita, 1997; Suito and Hirahara, 1999; Hyodo and Hirahara, 2004). In this study, we have used a parallelized finite element code GeoFEM developed at the Research Organization for Information Science and Technology (RIST) (e.g., Iizuka et al., 2002).

We set the model region as shown in Fig. 1, which includes the Tohoku and Hokkaido, northeastern Japan; the dimension of model region is approximately $1400 \mathrm{~km}$ (in $\sim \mathrm{EW}$ direction $) \times 1200 \mathrm{~km}$ (in $\sim \mathrm{NS}$ direction $) \times 200 \mathrm{~km}$ (depth). We did not take the curvature of the Earth's surface into account, so that the model surface is treated as a flat plane. The subsurface structure assigned to this model is shown in Fig. 2(a), which consists of four subregions, namely UC, LC, UM and PL. In making this subsurface structural model, we adopted the iso-depth contours of the Conrad and Moho planes given by Zhao et al. $(1992,1994)$. We also made use of the iso-depth contours of the upper plane of the Pacific plate drawn by Hagiwara (1986) (beneath Honshu area) and by Katsumata et al. (2003) (beneath Hokkaido area).

The model region was divided into a number of hexahedron elements to build a 3-D finite element grid (Fig. 2(b)). In this process, we employed a suit of utilities, called the CHIKAKU modeling system, including the CHIKAKUDB and CHIKAKU-CAD developed at the RIKEN (e.g., Kanai et al., 1999, 2000, 2001) and the CHIKAKU-MESH developed at the Japan Atomic Energy Agency (JAEA) (e.g., Miyamura et al., 2004; Oishi et al., 2004). The numbers of nodes and elements included in the finite element grid are 151,040 and 142,506, respectively. The typical element size near surface in the central portion of the grid is $\sim 15 \mathrm{~km}$ (in the horizontal direction) $\times 2 \mathrm{~km}$ (in the depth direction).

By using this 3-D finite element grid, in order to quantitatively estimate the effect of inhomogeneity of subsurface structure, we examine in detail the surface displace- 

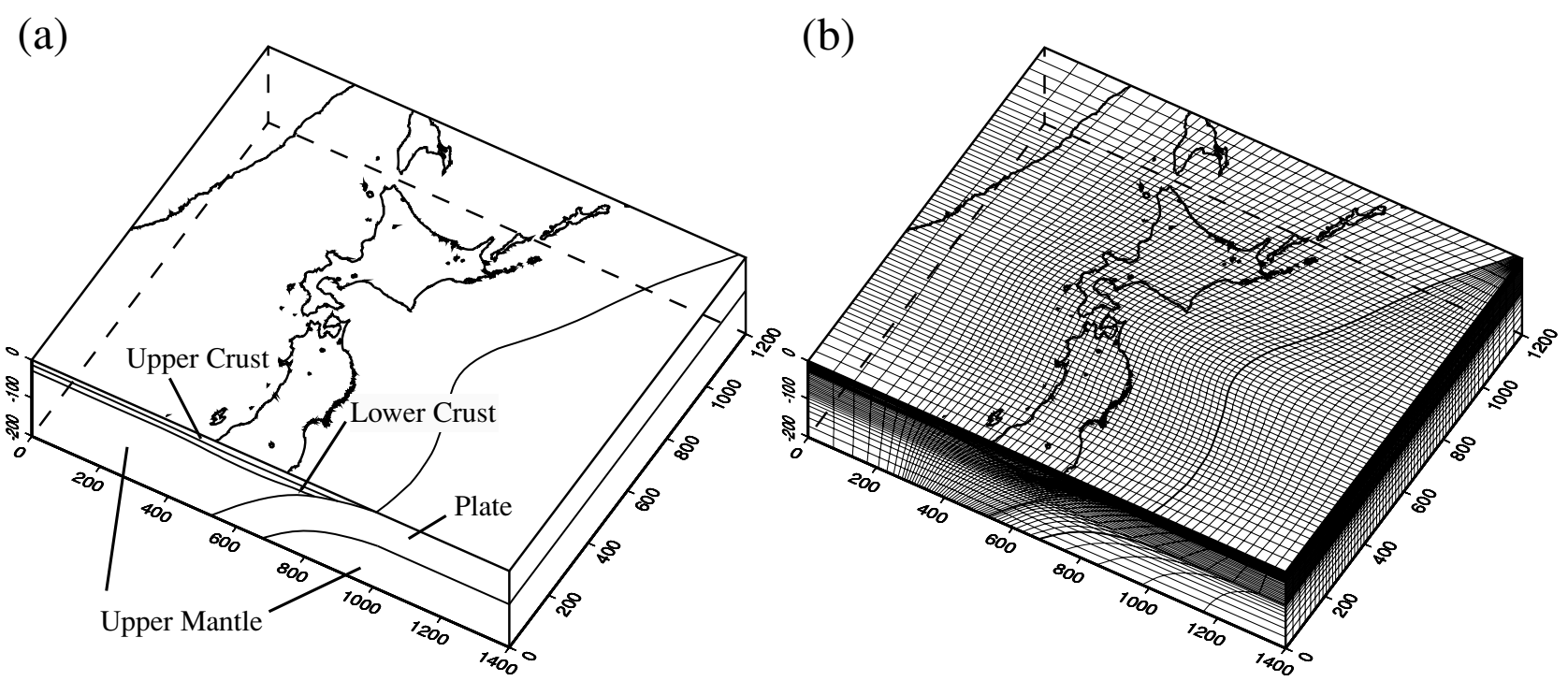

Fig. 2. (a) Outline of the 3-D model space with assumed subsurface structure. The model consists of four subregions, i.e., upper crust, lower crust, upper mantle and plate. (b) 3-D finite element grid with 151,040 nodes and 142,506 elements.

ments calculated with a variety of combinations of the elastic properties (i.e., the Young's modulus and Poisson's ratio) for UC, LC, UM and PL. We assume a set of cell-like subfaults distributed on the upper surface of the subducted Pacific plate in a depth range of about $8-100 \mathrm{~km}$ off and beneath Hokkaido. The number of these cell-like subfaults is 672 , and in the calculation we imposed independently on each of the cell-like subfaults a constant unit dislocation (i.e., $1 \mathrm{~m}$ reverse type dislocation toward an azimuth of 130 degrees). We incorporated the so-called "split node technique" in the finite element method for imposing such dislocation on each subfault. Figures 3(a) and (b) illustrate the distributed cell-like subfaults and the dislocation vectors on each of the subfaults superposed on the finite element grid along the upper boundary of the slab of the Pacific plate. In these figures, the cell-like subfaults and the dislocation vectors together with the finite element grid are all projected onto the model surface. The reason for calculating in this paper the surface displacements due to the unit dislocations on such cell-like subfaults is that we want to examine the effect of inhomogeneity of subsurface structure in the actual cases where the GF's necessary for inversions of the afterslip distribution on these subfaults following the 2003 Tokachi-oki earthquake are calculated with realistic setting. Examples of the vertical cross-sectional views (in $\sim$ EW direction) of subsurface structure and finite element grid are respectively shown in Figs. 4(a) and (b), which correspond to those for the southern end of the region on which the dislocations are imposed (the line A-B in Fig. 3(a)). The boundary conditions are as follows: we impose the socalled roller-conditions on the five model surfaces (i.e., the bottom surface and four side surfaces) except for the upper surface. In the roller-condition, the plane can move only in the tangential direction, but not to the normal direction, to the plane. The upper surface is assumed to be a free surface (i.e. a surface of stress free).

Next, description will be given for the elastic material parameters assigned to UC, LC, UM and PL. The subsurface structure beneath the northeastern Japan has been studied with seismological method by many authors. Among them, seismological tomography by Nakajima et al. (2001) is one of the recent ones. The 3 -D structures of the $P$ - and $S$-wave velocities derived by them have been used together with the densities given by Dambara and Tomoda (1969) to calculate the Young's modulus and Poisson's ratio of UC, LC and UM. We summarize in Table 1 the $P$ - and $S$-wave velocities, densities and resultant Young's modulus and Poisson's ratio of UC, LC and UM. On the other hand, we adopted the Young's modulus and Poisson's ratio of PL respectively given by Liu (1980) and Suito and Hirahara (1999), which are also presented in Table 1.

In order to accomplish our purpose of this paper, that is to quantitatively clarify how much the inhomogeneity of subsurface structure affects on the displacements at surface, we consider several models with various settings of the elastic material parameters for UC, LC, UM and PL other than those given in Table 1. The models considered in the present study are listed in Table 2. The models are classified into two categories, namely homogeneous elastic model (HE-model) and inhomogeneous elastic model (IEmodel) groups. The HE-model group includes three models, i.e., HE-1, HE-2 and HE-3; whereas the IE-model group includes four models, i.e., IE-1, IE-2, IE-3 and IE-4. A detailed model description will be given below.

The entire model space of HE-1, HE-2 and HE-3 is homogeneous so that they have no subsurface structure. The displacements and strains due to dislocations within a homogeneous elastic material depend only on the Poisson's ratio but not on the Young's modulus. Hence, in our three homogeneous models, the Young's modulus is the same, but the Poisson's ratio is different from each other. HE-1 is assigned a standard value of 0.25 as the Poisson's ratio, whereas HE-2 and HE-3 are assigned respectively smaller or larger value by 0.02 than 0.25 .

Next, the material parameters for inhomogeneous models are as follows. IE- 1 is a basic inhomogeneous model for 
(a)

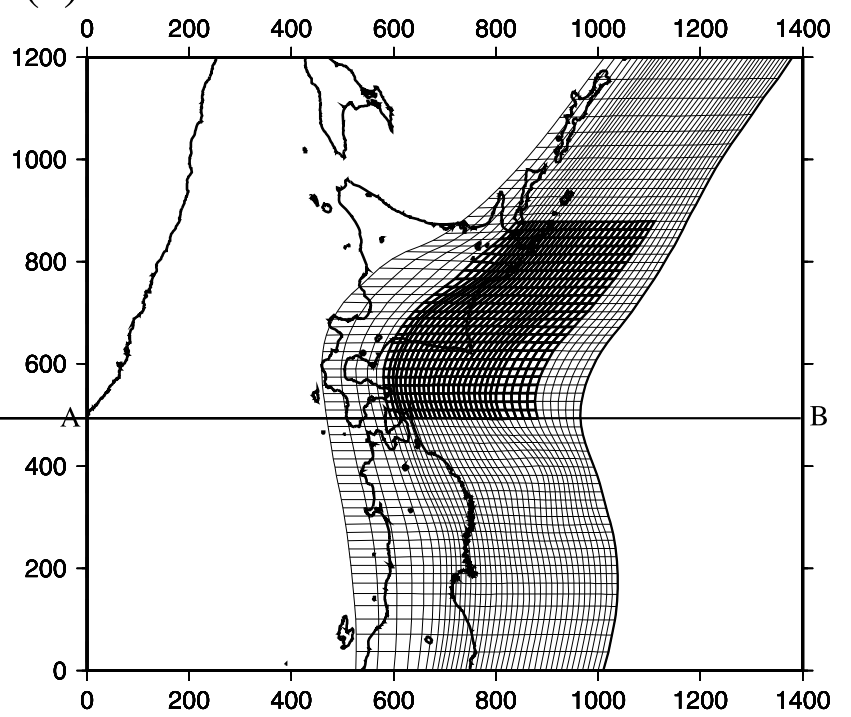

(b)

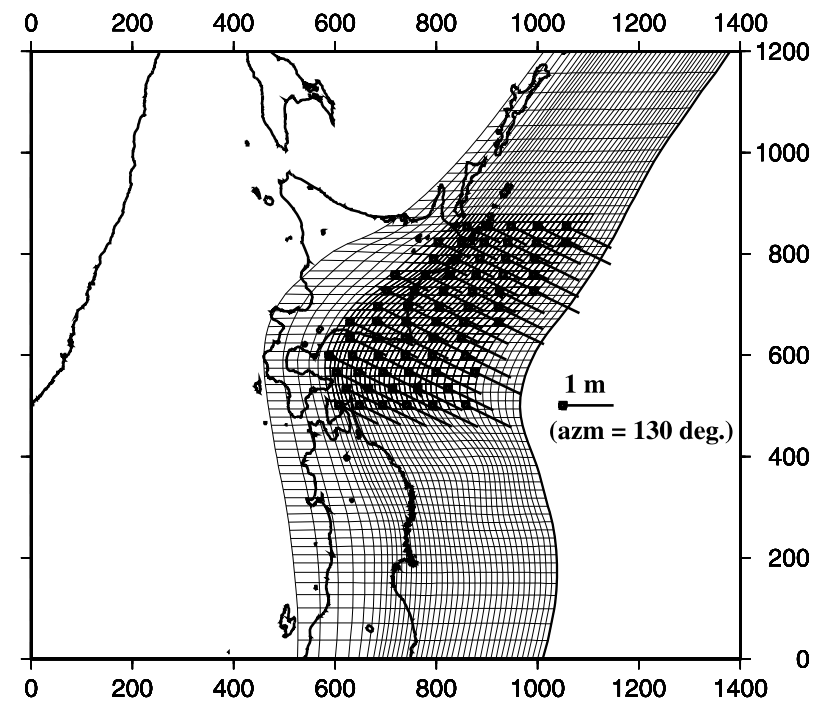

Fig. 3. (a) Cell-like subfaults assumed on the subducted Pacific plate interface. The subfaults are distributed in a depth range of approximately $8-100 \mathrm{~km}$, and the number of them is $672(=24 \times 28)$. The grid shown is the one for the Pacific plate interface and projected onto the model surface. (b) Vectors of $1 \mathrm{~m}$ reverse type dislocation imposed independently on each of the cell-like subfaults. Note that the vectors are shown also as those projected onto the model surface and, for simplicity, on each five subfaults.
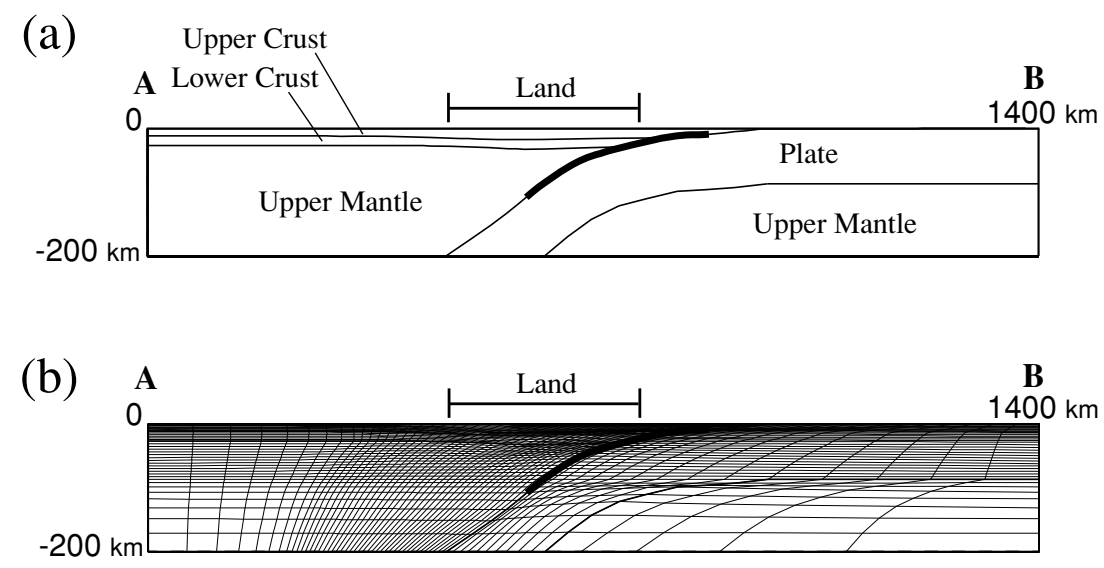

Fig. 4. Examples of vertical cross-sectional views (in $\sim$ EW direction) for (a) subsurface structure and (b) finite element grid. These figures correspond to the line A-B in Fig. 3(a), and the thick lines along the Pacific plate interface indicate the portion on which the dislocations are imposed.

Table 1. $V_{\mathrm{p}}, V_{\mathrm{s}}$, densities and elastic material parameters.

\begin{tabular}{|c|c|c|c|c|c|}
\hline Region & $V_{\mathrm{p}}{ }^{a}$ & $V_{\mathrm{s}}^{b}$ & $\rho^{c}$ & $E^{d}$ & $v^{e}$ \\
\hline Upper Crust & 5.664 & 3.300 & 2.67 & 72.3 & 0.243 \\
\hline Lower Crust & 6.570 & 3.730 & 3.00 & 105 & 0.263 \\
\hline Upper Mantle & 8.270 & 4.535 & 3.32 & 176 & 0.285 \\
\hline Plate & - & - & - & 95.4 & 0.258 \\
\hline \multicolumn{2}{|c|}{${ }^{a} P$-wave velocity $(\mathrm{km} / \mathrm{s})$} & \multicolumn{4}{|c|}{ for Upper Crust, Lower Crust and Upper Mantle } \\
\hline \multicolumn{2}{|c|}{${ }^{b} S$-wave velocity $(\mathrm{km} / \mathrm{s})$} & & \multicolumn{3}{|c|}{$V_{\mathrm{p}}, V_{\mathrm{s}}:$ Nakajima et al. (2001) } \\
\hline \multicolumn{2}{|l|}{${ }^{c}$ density $\left(\mathrm{g} / \mathrm{cm}^{3}\right)$} & & \multicolumn{3}{|c|}{$\rho:$ Dambara and Tomoda (1969) } \\
\hline \multicolumn{2}{|c|}{${ }^{d}$ Young's modulus (GPa) } & for Plate & & & \\
\hline \multirow{2}{*}{\multicolumn{2}{|c|}{${ }^{e}$ Poisson's ratio }} & & \multicolumn{3}{|c|}{$E$ : Liu (1980) } \\
\hline & & & \multicolumn{3}{|c|}{$v$ : Suito and Hirahara (1999) } \\
\hline
\end{tabular}

which the material parameters given in Table 1 are assigned. Young's modulus of IE-2). On the other hand, IE-3 has the IE-2 has the same Poisson's ratios as IE-1 for UC, LC, UM same Young's moduli as IE-1 for all of the portions, but and PL, but the Young's moduli for all of these portions the Poisson's ratios are unified to the same value of 0.25 are assigned same value of $100 \mathrm{GPa}$ (i.e., no contrast in the for entire of the model space. Moreover, we consider one 
Table 2. Model descriptions with material parameter settings.

\begin{tabular}{|c|c|c|c|c|c|c|c|c|}
\hline \multirow[b]{2}{*}{ Model } & \multicolumn{4}{|c|}{ Young's modulus (GPa) } & \multicolumn{4}{|c|}{ Poisson's ratio } \\
\hline & $\mathrm{UC}^{a}$ & $\mathrm{LC}^{b}$ & $\mathrm{UM}^{c}$ & $\mathrm{PL}^{d}$ & $\mathrm{UC}^{a}$ & $\mathrm{LC}^{b}$ & $\mathrm{UM}^{c}$ & $\mathrm{PL}^{d}$ \\
\hline HE-1 & 100 & 100 & 100 & 100 & 0.250 & 0.250 & 0.250 & 0.250 \\
\hline HE-2 & 100 & 100 & 100 & 100 & 0.230 & 0.230 & 0.230 & 0.230 \\
\hline HE-3 & 100 & 100 & 100 & 100 & 0.270 & 0.270 & 0.270 & 0.270 \\
\hline IE-1 & 72.3 & 105 & 176 & 95.4 & 0.243 & 0.263 & 0.285 & 0.258 \\
\hline IE-2 & 100 & 100 & 100 & 100 & 0.243 & 0.263 & 0.285 & 0.258 \\
\hline IE-3 & 72.3 & 105 & 176 & 95.4 & 0.250 & 0.250 & 0.250 & 0.250 \\
\hline IE-4 & 72.3 & 105 & 100 & 95.4 & 0.243 & 0.263 & 0.285 & 0.258 \\
\hline
\end{tabular}

more inhomogeneous model IE-4 in order to estimate the influence of the Young's modulus of UM, whose volume is much larger than those of UC, LC and PL, on the surface displacements. In this model, the Young's modulus of UM is set to $100 \mathrm{GPa}$, which is approximately half of that in IE1 (i.e., $176 \mathrm{GPa}$ ), and those of UC, LC and PL are the same as those in IE-1 (the Poisson's ratios for UC, LC, UM and $\mathrm{PL}$ are also the same as those in IE-1).

\section{Results}

As described in the previous section, we calculate and compare the displacements on surface for the seven models (i.e., HE-1, HE-2, HE-3, IE-1, IE-2, IE-3 and IE-4) due to the dislocation independently given to each of the 672 celllike subfaults on the plate interface. Using the displacement vectors at the nodes on the surface of the finite element grid, we recalculate the displacement vectors at 144 positions, i.e., the positions of 142 GEONET stations in the northern Tohoku district and Hokkaido and two ocean-bottom pressure gauges operated by JAMSTEC (e.g., Hirata et al., 2002). Then, in order to evaluate numerically the differences in the surface displacements between models, we calculate the RMS discrepancies in the 144 surface displacements due to the 672 independent dislocations between each pair of the seven models. The RMS discrepancy $\epsilon$ between, for example, models A and B is calculated from

$$
\begin{aligned}
\epsilon= & {\left[\frac { 1 } { N _ { p } N _ { f } } \sum _ { i = 1 } ^ { N _ { p } } \sum _ { j = 1 } ^ { N _ { f } } \left\{\left(U_{A, i j}-U_{B, i j}\right)^{2}\right.\right.} \\
& \left.\left.\quad+\left(V_{A, i j}-V_{B, i j}\right)^{2}+\left(W_{A, i j}-W_{B, i j}\right)^{2}\right\}\right]^{1 / 2},
\end{aligned}
$$

where $(U, V, W)$ is the $x$-, $y$ - and $z$-components of the displacement vector at each position, and suffixes $A$ and $B$ respectively correspond to models $\mathrm{A}$ and $\mathrm{B}$. Suffixes $i$ and $j$ respectively correspond to the serial numbers of positions and cell-like subfaults, and $N_{p}$ and $N_{f}$ respectively mean the numbers of positions (i.e., 144) and cell-like subfaults (i.e., 672).

Thus calculated values of the RMS discrepancy $\epsilon$ for each model pair are tabulated in Table 3 (note that, for comparison, we also presented in this table the RMS discrepancies for each model pair in the case where only 288 subfaults in the seismogenic depth range of about 20-60 $\mathrm{km}$, instead of 672 subfaults in the depth range of about 8-100 km, are considered). In the table, very small values of $\epsilon$ (i.e., less than $0.01 \mathrm{~cm}$ ) are presented in parentheses. From the table, we find some points as follows (they are valid for the both cases where the subfaults in the depth range of about $8-100 \mathrm{~km}$ are considered and where those only in the seismogenic depth range of about $20-60 \mathrm{~km}$ are considered). First, the values of $\epsilon$ for the pairs among the homogeneous models (i.e., HE-1, HE-2 and HE-3) are very small so that we can say no meaningful discrepancy in the surface displacements exists among these models. These three models have the same Young's modulus but the Poisson's ratios are different from each other by $0.02(0.25$ in HE-1, 0.23 in HE-2, and 0.27 in HE-3). Next, it is also found that the discrepancies in the surface displacements among the homogeneous and inhomogeneous models are large for some model pairs and very small for other ones. The pairs with small discrepancy are between each homogeneous model and an inhomogeneous model IE-2. On the other hand, those with large discrepancy are between each homogeneous model and the rest of inhomogeneous models, i.e., IE-1, IE-3 and IE-4. Although the inhomogeneous model IE-2 that shows small difference between homogeneous models has different Poisson's ratios for UC, LC, UM and PL, it has homogeneous Young's modulus of $100 \mathrm{GPa}$. The fact that this inhomogeneous model does not give rise to large discrepancy between homogeneous models means that the contrast in the Poisson's ratio between model subregions (i.e., UC, LC, UM and PL) gives little influence on the surface displacements. On the contrary, the inhomogeneous models IE-1, IE-3 and IE-4 which show large discrepancy between homogeneous models have different Young's moduli for UC, LC, UM and PL, while the Poisson's ratios are different between these subregions in some models but same in other models. Finally, we find that the discrepancies in the surface displacements among the inhomogeneous models are generally large with one exception. The exception with little difference in the surface displacements is the model pair (IE-1 and IE-3). The Young's moduli for UC, LC, UM and PL are equal between the models IE-1 and IE-3, and only the Poisson's ratios for these subregions are different between these two models. All of these points described above mean that the surface displacements do not much depend on the Poisson's ratio, but much depend on the Young's modulus. In other words, the Young's modulus or its contrast within models much affects on the surface displacements than the Poisson's ratio or its contrast. The reason for this will be discussed later. We should, however, emphasize here that it is of course only in the 
Table 3. RMS discrepancies between model pairs (unit: $\mathrm{cm}$ ).

\begin{tabular}{ccccccc}
\hline Model & HE-2 & HE-3 & IE-1 & IE-2 & IE-3 & IE-4 \\
\hline HE-1 & $(0.002)$ & $(0.002)$ & 0.029 & $(0.003)$ & 0.028 & 0.017 \\
& $(0.002)$ & $(0.003)$ & 0.026 & $(0.003)$ & 0.025 & 0.014 \\
HE-2 & & $(0.005)$ & 0.029 & $(0.004)$ & 0.028 & 0.017 \\
& & $(0.005)$ & 0.026 & $(0.006)$ & 0.025 & 0.014 \\
HE-3 & & 0.029 & $(0.003)$ & 0.028 & 0.017 \\
& & & 0.026 & $(0.003)$ & 0.025 & 0.014 \\
IE-1 & & & 0.028 & $(0.003)$ & 0.020 \\
& & & & 0.025 & $(0.003)$ & 0.025 \\
IE-2 & & & & 0.028 & 0.016 \\
& & & & & 0.024 & 0.012 \\
IE-3 & & & & & 0.020 \\
& & & & & & 0.025 \\
\hline
\end{tabular}

Note: values in upper rows and lower rows are respectively the RMS discrepancies in the cases where the subfaults in the depth ranges of about 8-100 km (i.e., 672 subfaults) and of about 20$60 \mathrm{~km}$ (i.e., 288 subfaults) are considered, and values in parentheses are negligibly small ones (i.e., less than 0.01).

case of inhomogeneous material that the Young's modulus much affects on the surface displacements (as mentioned already, the displacements due to dislocations within a homogeneous material depend only on the Poisson's ratio but not on the Young's modulus).

Then we compare the surface displacement vectors between the model pairs of large discrepancies in the displacements. We choose the model pair (HE-1 and IE-1) as a representative example of such ones, and plot the horizontal surface displacement vectors for this model pair in Fig. 5(a). The surface displacements shown in the figure are those caused by the dislocation at the cell-like subfault indicated by a square in the figure. It can be seen in the figure that, although the directions of horizontal surface displacement vectors in the inhomogeneous model IE-1 are not much different from those in the homogeneous model HE-1 (the maximum discrepancy in the directions of horizontal surface displacements between these two models is $\sim 15^{\circ}$ and most of them are less than $10^{\circ}$ ), the magnitudes of them are significantly smaller than those in the homogeneous model. Next, comparison of the vertical surface displacements for the same model pair is shown in Fig. 5(b). It is found that the vertical surface displacements in the inhomogeneous model IE-1 are also slightly smaller than those in the homogeneous model HE-1 at most positions in the land area. On the contrary, in the ocean area, those in the homogeneous model are smaller than those in the inhomogeneous model.

In order to see the dependence of surface displacements in HE-1 and IE-1 and that of their differences on the distance from the trench, we show in Figs. 6(a), (b) and (c) the profiles of total, horizontal and vertical surface displacements in these models along a line in SE-NW direction shown in Figs. 5(a) and (b) as line X-Y. It is noticeable that the total and horizontal surface displacements in HE-1 are larger than those in IE-1 at any point along the profile line. These displacements in both models gradually increase with distance from the trench and take their maximum values near the position above the subfault on which the dislocation is imposed (with an approximate distance of $200 \mathrm{~km}$ from the trench). Beyond this position toward land area they rapidly decrease with distance up to $\sim 400 \mathrm{~km}$ and then decrease gradually. Their differences also become maximum value near the position above the subfault on which the dislocation is imposed. On the other hand, vertical displacement profiles show somewhat complex behavior. Vertical displacements both in HE-1 and IE1 are negative (i.e., down-ward) in the distance range from 0 to $\sim 120 \mathrm{~km}$ and positive (i.e., up-ward) in the distance range between $\sim 120 \mathrm{~km}$ and $\sim 530 \mathrm{~km}$. Beyond the position of a distance of $\sim 530 \mathrm{~km}$, both of them again become negative. However, absolute values of the vertical displacement in IE-1 is always larger than that in HE-1 in the distance range between 0 and $\sim 280 \mathrm{~km}$, while beyond this position the latter is larger than the former. The difference in the vertical displacements in HE-1 and IE-1 is larger near the trench. Although we do not show figures, it can be qualitatively said that such characteristics in the surface displacements are almost commonly found irrespective of the position of subfault on which the dislocation is imposed. Furthermore, these characteristics are almost similarly recognized for other model pairs of large discrepancies in the surface displacements such as (HE-2 and IE-1), (HE-3 and IE-1), (HE-1 and IE-3), (HE-2 and IE-3) and (HE-3 and IE3).

Thus we can say that the surface displacements in the inhomogeneous models are generally smaller than those in the homogeneous models. In order to quantitatively clarify the spatial distributions of the discrepancies in the surface displacements between the homogeneous and inhomogeneous models, we plot them in Fig. 7. This figure shows such discrepancy between, as an example, HE-1 and IE- 1 in the surface displacements caused by a dislocation on the same cell-like subfault as that shown in Figs. 5(a) and (b). In the figure, we show the discrepancies of surface displacements in IE-1 from those in HE-1 as to the magnitudes of total displacements, horizontal displacements, vertical displacements, and the directions of total displacement vectors. Since the relative discrepancies seem to be more meaningful than the absolute ones as to the magnitudes of discrepancies, the discrepancies shown in Figs. 7(a), (b) and (c) are the relative discrepancies calculated by $\{$ (displacements 


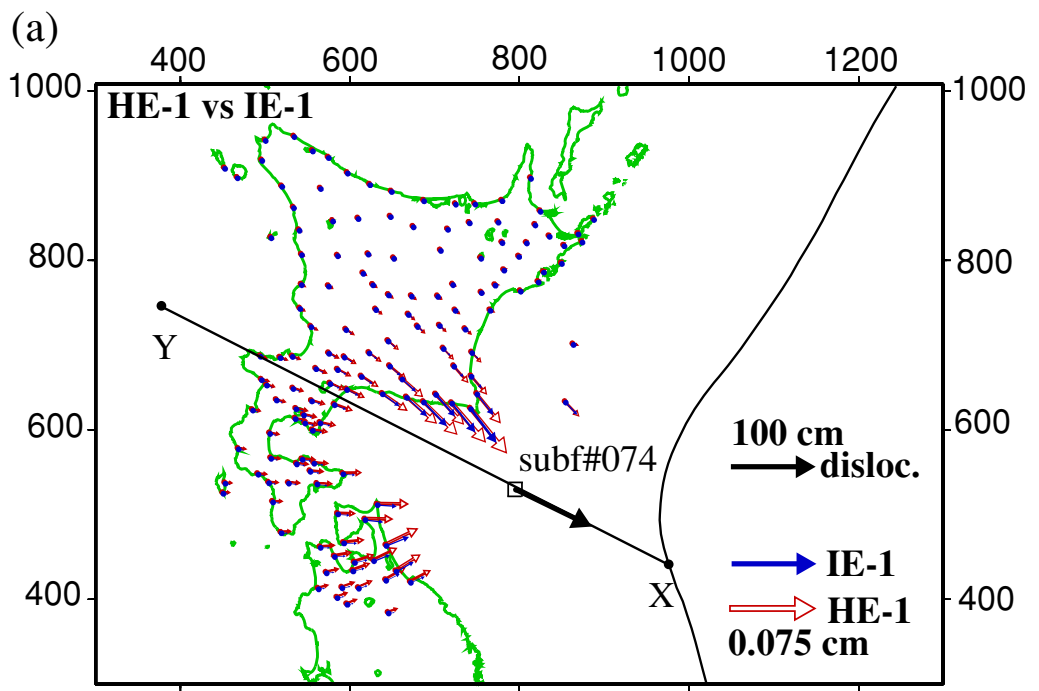

(b)

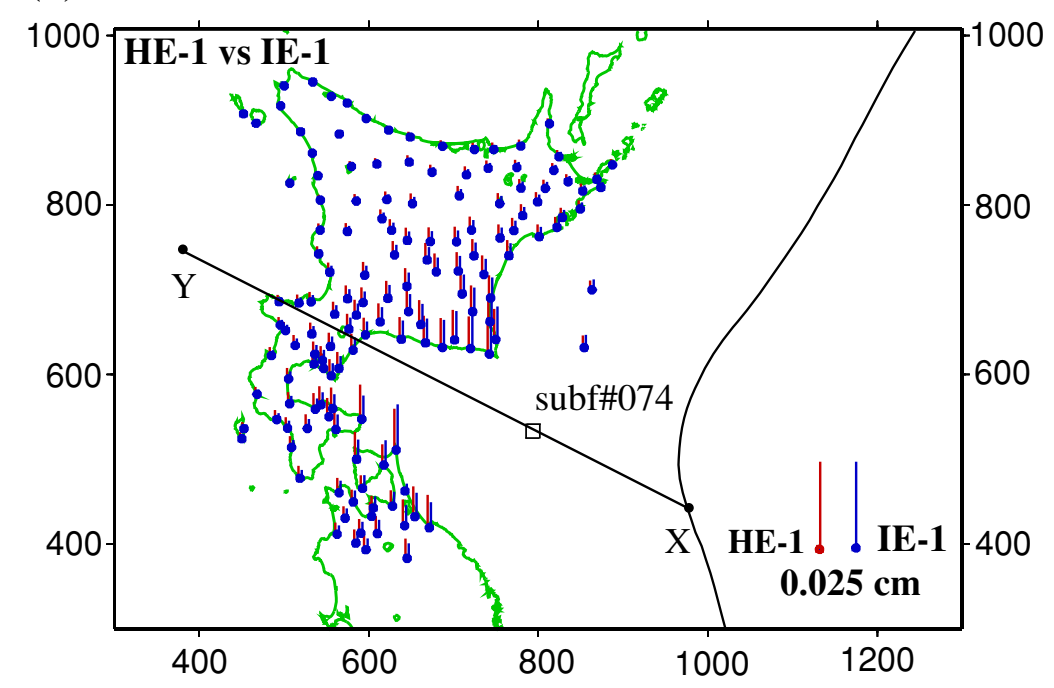

Fig. 5. Comparison of the surface displacements between homogeneous model HE-1 and inhomogeneous model IE-1. (a) Horizontal displacement vectors, and (b) vertical displacements. These figures show the surface displacements due to the reverse type dislocation of $1 \mathrm{~m}$ imposed on the cell-like subfault indicated in each figure. The line $\mathrm{X}-\mathrm{Y}$ in each figure is that along which the surface displacement profiles are shown in Figs. 6(a), (b) and (c).

(a) Total Displacement

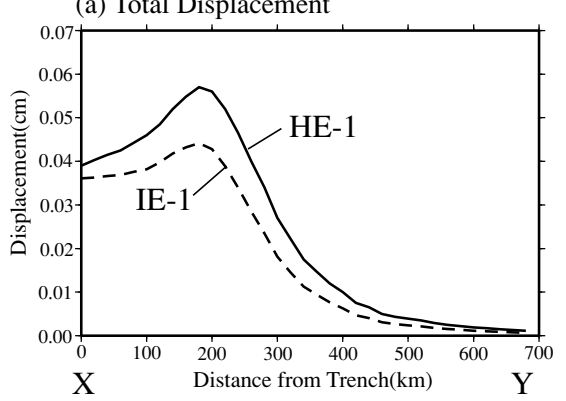

(b) Horizontal Displacement

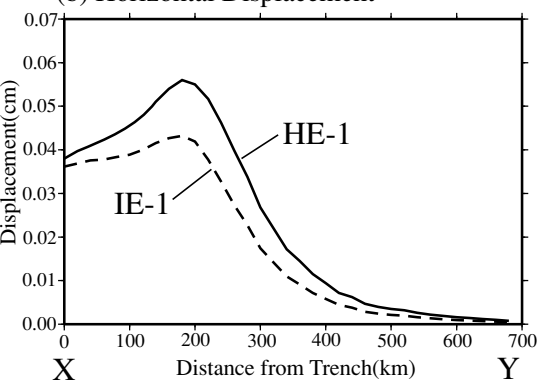

(c) Vertical Displacement

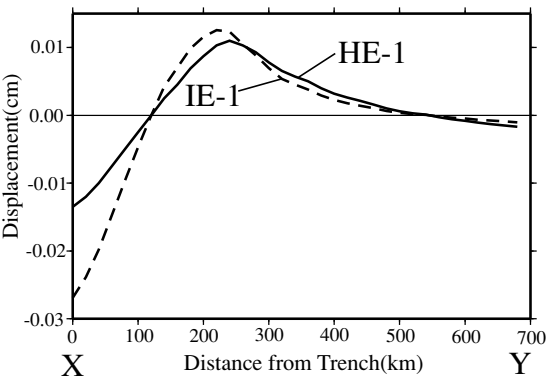

Fig. 6. Profiles of (a) total, (b) horizontal and (c) vertical surface displacements along the line X-Y shown in Figs. 5 (a) and (b). Profiles for both HE-1 and IE-1 are shown in each figure.

in IE-1) - (displacements in HE-1) $\}$ / (displacements in HE-1) (polarities are also considered for the vertical displacements so that up- and down-ward displacements are respectively treated as positive and negative values). For reference, in these figures the contours respectively for the distribution of total, horizontal and vertical displacements (in $\mathrm{cm}$ ) in HE-1 are also shown. In Fig. 7(d), the discrepancies in the directions are shown as the angles between the displacement vectors in IE-1 and those in HE-1. Note that we do not show in the figures the discrepancies and contours 
(a) Total Displacement

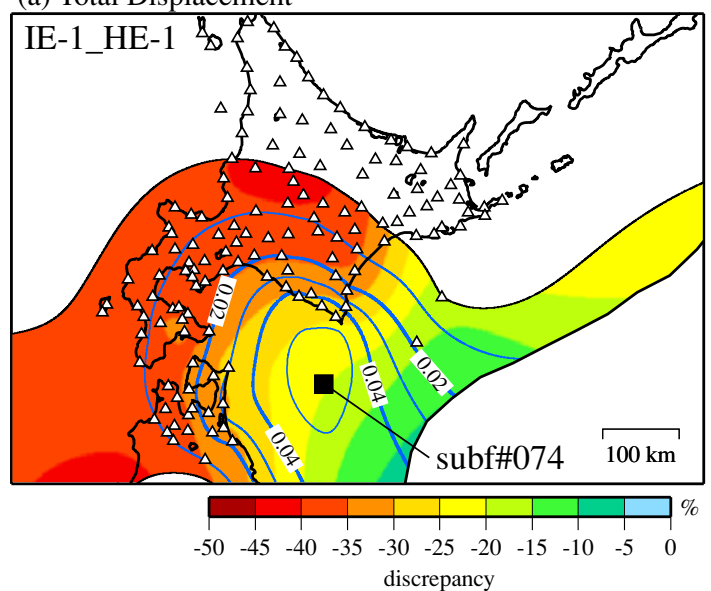

(c) Vertical Displacement

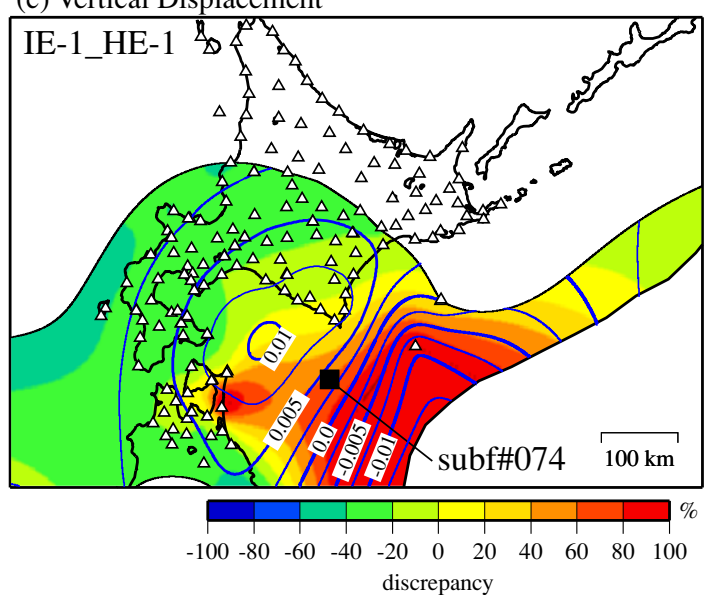

(b) Horizontal Displacement

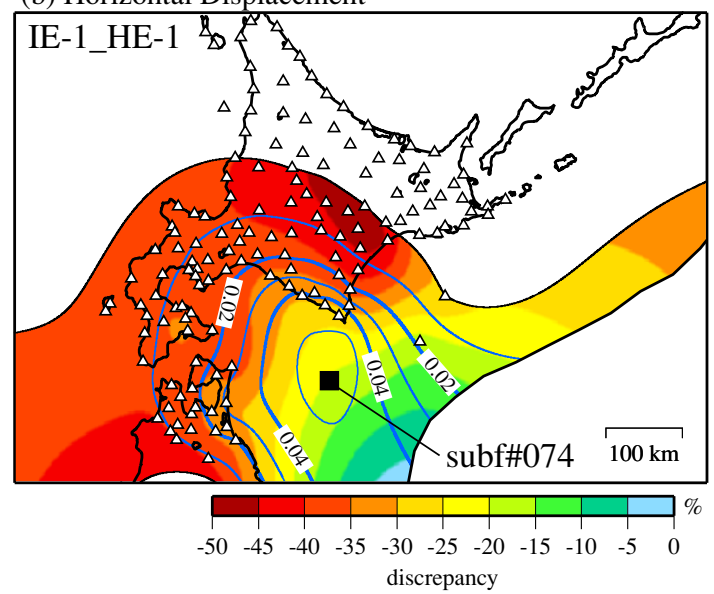

(d) Direction of Total Displacement

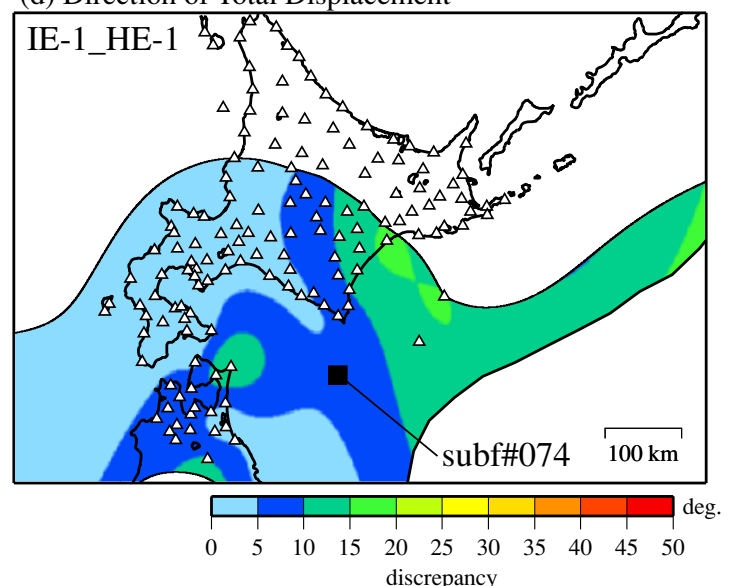

Fig. 7. Spatial distribution of the discrepancies in the surface displacements due to the dislocation on the same subfault as that in Figs. 5(a) and 5(b) between IE-1 and HE-1. Four figures respectively correspond to the discrepancies in (a) magnitudes of total displacements, (b) magnitudes of horizontal displacements, (c) magnitudes of vertical displacements, and (d) directions of the total displacements. The discrepancies in the magnitudes shown are the values calculated by $\{$ (displacements in IE-1) - (displacements in HE-1) $\}$ / (displacements in HE-1) (polarities are also considered for the vertical displacements so that up- and down-ward displacements are respectively treated as positive and negative values), whereas the discrepancies in the directions are shown as the angles between the displacement vectors in IE-1 and those in HE-1. Contours in (a), (b) and (c) respectively show the distribution of total, horizontal and vertical displacements (in $\mathrm{cm}$ ) in HE-1. Note that in each figure the discrepancies and contours are not shown for the region where the total surface displacements in HE-1 are less than $10 \%$ of the maximum ones. Triangles indicate the positions for which the horizontal and vertical displacements are plotted in Fig. 5.

for the region where the total surface displacements in HE1 are less than $10 \%$ of the maximum ones, since comparing such negligibly small displacements between the models seems less meaningful. Some points as follows can be realized from these figures. First, the spatial distributions of discrepancies in the total and horizontal surface displacements are similar to each other; the discrepancies become larger gradually from the trench region toward land area. The discrepancies are $\sim 30 \%$ in the Pacific coast region, and they reach $\sim 40 \%$ at some portion of inland area. On the other hand, discrepancies in the vertical surface displacements are smallest at near the Cape Erimo, Pacific coast of Hokkaido, and become larger toward the trench region and toward the inland area to Japan Sea coast. The maximum discrepancy in the vertical displacements reaches $\sim 100 \%$ near the trench. Note that the discrepancies in the vertical displacements in the Pacific coast area are positive (i.e., absolute values of the vertical displacements in the inhomogeneous model IE-1 are larger than those in the homogeneous model HE-1) while those in the inland to Japan Sea coast areas are negative (i.e., absolute values of the vertical displacements in IE-1 are smaller than those in HE-1). The angles between the surface displacement vectors in IE-1 and HE-1 are not very large; they are less than $10^{\circ}$ in most area and $\sim 15^{\circ}$ at maximum. Hence, the directions of surface displacements in the inhomogeneous and homogeneous models are not much different from each other. In a qualitative sense, it can be said that such characteristics of the discrepancies in the surface displacements between the inhomogeneous and homogeneous models does not depend on the position of the subfault on which the dislocation is imposed. However, in a quantitative sense, there are some differences between the cases where the dislocations are imposed on the subfaults in different areas. For instance, if the dislocation is imposed on the subfaults near the trench, the discrepancies in the total or horizontal surface displacements are $30-40 \%$ in the Pacific coast region with more than $40 \%$ in some of inland area, while they are $\sim 10 \%$ near the trench region. On the contrary, if the dislocation is imposed on the subfaults near or beneath the land area, those are $\sim 10 \%$ in 
the Pacific coast region, whereas those are $30-40 \%$ in the trench region. Thus, roughly speaking, the discrepancies in the surface displacements between the inhomogeneous and homogeneous models are larger in the far field and smaller in the near field.

\section{Discussion}

It has been found as described in the previous section that the surface displacements in inhomogeneous models are significantly smaller than those in homogeneous models by $\sim 30 \%$ in most region and more than $40 \%$ at maximum. Such discrepancies in the surface displacements generally depend on the distance from the source, i.e., the position of subfault on which the dislocation is imposed. Moreover, as the result of detailed examination of the effect of inhomogeneities in the Young's modulus and Poisson's ratio on the surface displacements with various models having different setting of these material parameters, it has also been found that inhomogeneity in the Young's modulus affects on the surface displacements more than that in the Poisson's ratio. In this section, some points on these results will be discussed.

First, it is understandable that the discrepancies in the surface displacements between the inhomogeneous and homogeneous models roughly depend on the distance from the source. The reason for this is that, since the effect of inhomogeneity (i.e., contrasts in the Young's modulus and/or Poisson's ratio between the subregions such as UC, LC, UM and PL) should be accumulated towards the calculation point from the source, it would be larger if the distance between the source and calculation point is larger.

Next, let us consider the reason why inhomogeneity in the Young's modulus affects more than that in the Poisson's ratio on the surface displacements caused by dislocations within the material. Dislocation is of course tangential or shearing motion on a fault, so that the deformation caused by it would much depend on the shear modulus or rigidity of the material. The shear modulus or rigidity $G$ can be expressed as $G=E / 2(1+v)$, where $E$ is the Young's modulus and $v$ is the Poisson's ratio. Therefore we obtain $(d G / G)=(d E / E)-\{v /(1+v)\}(d v / v)$. If we put 0.25 as a typical value for the Poisson's ratio $v$, this equation becomes $(d G / G)=(d E / E)-0.2(d v / v)$. This means that the same amount of perturbation in the Young's modulus $E$ (i.e., $d E / E$ ) can give rise to five times larger perturbation in the shear modulus or rigidity $G$ (i.e., $d G / G$ ) than that in the Poisson's ratio (i.e., $d v / v$ ). Hence it is reasonable that inhomogeneity in the Young's modulus affects more than that in the Poisson's ratio on the deformation caused by dislocations. Therefore, in order to obtain accurate estimation of surface displacements due to dislocations, the Young's modulus for subsurface materials should be more accurately known than the Poisson's ratio. However, it is unfortunately more difficult to determine the Young's modulus than to determine the Poisson's ratio, since the latter can be derived from the $P$ - and $S$-wave velocities of the material which are well constrained through seismic studies while determining the former requires an additional parameter, that is, the density, for which detailed information is not sufficiently obtained. Thus it is desirable to study the distribution of densities for the subsurface structure as well as those of the seismic velocities.

By the way, there might be a doubt if these results are only apparent ones caused by the prescribed boundary conditions in the finite element calculations. In order to test this, some additional calculations with different boundary conditions have also been accomplished. In these calculations, we remove the so-called roller-conditions imposed on the bottom surface and four side surfaces of the model space. Instead, we constrain the four apexes included in the bottom surface out of eight apexes of the model space not to move horizontally and vertically, and the remaining four apexes included in the model surface not to move only horizontally. The surface displacements thus calculated show little difference from those with the roller-conditions except for those near the model boundaries. Hence, it is found that the roller-conditions do not much disturb the surface displacements in most area, so that the effect of the rollerconditions is negligibly small. Therefore, the discrepancies in the surface displacements between the homogeneous and inhomogeneous models presented in the previous section are not apparent ones due to the imposed boundary conditions.

Finally, we compare our results on the discrepancies in the surface displacements between homogeneous and inhomogeneous models with those in other similar studies. Here we pick up the studies by Wald and Graves (2001) and Masterlark (2003), mentioned earlier, as the examples of such studies and compare our results with theirs. Wald and Graves (2001) calculated and compared the surface displacements due to some distributed dislocations on a subsurface fault in the San Fernando basin region, California, with assumptions of homogeneous and inhomogeneous elastic materials. They found from the calculations that there are very large discrepancies of $\sim 30 \%$ at maximum in the surface displacements between the cases of homogeneous and inhomogeneous materials. Although these discrepancies are somewhat smaller than those in the present study, their results also indicate that there should be very large differences in the surface displacements between homogeneous and inhomogeneous elastic materials. The results by Masterlark (2003), which were obtained for the surface displacements caused by dislocations distributed on the plate interface in a subduction zone off Mexico, indicated quite large $(\sim 100 \%$ in some portion of his model space) differences in the surface displacements between homogeneous and inhomogeneous elastic materials. This amount of differences are much larger than those in the present study, suggesting that in subduction zones the subsurface inhomogeneities could give rise to significantly large discrepancies in the surface displacements.

In the present study, we intended to emphasize that there would be much discrepancies in the surface displacements between homogeneous and inhomogeneous cases. At the same time, we also intended to elucidate the effects of the elastic properties such as the Young's modulus and Poisson's ratio on the surface displacements. Hence we assigned artificial values to these elastic properties in the models except for IE-1, because it would be better to use much different values as these parameters in different models in 
order to clearly show the effects of these parameters. It might be also important, and thus worthwhile in the future, to evaluate the effects of uncertainties in these elastic parameters, which are derived from the seismic velocities and densities, on the surface displacements.

The results obtained in this study that the surface displacements are much different from each other between homogeneous and inhomogeneous models and that those in homogeneous models are larger than those in inhomogeneous models by $20-30 \%$ with about $40 \%$ at maximum will give rise to an important influence. They mean that there should be a possibility that the dislocations on subsurface faults estimated by using GF's calculated for homogeneous elastic material are significantly smaller than actual ones. Hence, in order to estimate more accurate distributions of the subsurface dislocations, it is important to use the GF's calculated with realistic subsurface structures with adequate material properties such as the Young's modulus and Poisson's ratio, instead of those for the homogeneous elastic half space mostly used so far. For instance, as mentioned in the first section, there have already been some inversion studies in which the distribution of coseismic slip or afterslip associated with the 2003 Tokachi-oki (Hokkaido, northeastern Japan) earthquake ( $M$ 8.0) was derived from the displacements at many sites of GEONET in Hokkaido and northern Tohoku which are the same regions as those modeled in this work (e.g., Miyazaki et al., 2004a, 2004b; Miura et al., 2004; Ozawa et al., 2004) or from the landbased GEONET data together with those by ocean-bottom pressure gauges (Baba et al., 2006). However, most of these studies used the GF's for the surface displacements calculated with the assumptions of homogeneous elastic half space. Therefore, in order to estimate more accurately the afterslip distribution, it may be necessary to reevaluate it by using the GF's calculated for the realistic inhomogeneous subsurface structure such as those obtained in the present study. Hence we are now proceeding to the calculation of the afterslip distribution following the 2003 Tokachi-oki earthquake by using the surface displacements obtained in the present study as the GF's. The results will be presented elsewhere.

\section{Conclusions}

In this study, we quantitatively evaluate the effect of inhomogeneity of subsurface structure on the surface displacements through numerical calculations by using a 3-D finite element grid for the northeastern Japan (i.e., the Tohoku and Hokkaido districts). The finite element grid has a size of $1400 \mathrm{~km}$ (in $\sim \mathrm{EW}$ direction) $\times 1200 \mathrm{~km}$ (in $\sim \mathrm{NS}$ direction) $\times 200 \mathrm{~km}$ (depth). We consider two types of models as to the material parameter settings (i.e., the Young's modulus and Poisson's ratio) for the subsurface structure; one is the homogeneous type having no subsurface structure, while another is the inhomogeneous one having some subsurface structure. The homogeneous and inhomogeneous types include respectively three and four models with various material parameter settings. We calculate and compare the surface displacements in each model caused by a unit dislocation on each of 672 cell-like subfaults distributed on the upper interface of the Pacific plate.
The results of calculations can be summarized as follows:

(1) The surface displacements in inhomogeneous models are significantly smaller than those in homogeneous models by $\sim 30 \%$ in most region with a maximum discrepancy of $\sim 40 \%$.

(2) Inhomogeneity in the Young's modulus affects on the surface displacements more than that in the Poisson's ratio.

Based on these results, we may conclude that using the GF's obtained for homogeneous elastic half space is not adequate, especially in the regions such as those in and around Japan where inhomogeneity of subsurface structure is large.

Acknowledgments. We thank all of the staff at the Institute for Research on Earth Evolution (IFREE), Japan Agency for MarineEarth Science and Technology (JAMSTEC), and Prof. Kazuro Hirahara at Kyoto University for their helpful discussions and advices. Comments from two anonymous reviewers were very useful to improve the manuscript. The 3-D finite element grid used in this study was created by using the CHIKAKU system including the CHIKAKU-DB, CHIKAKU-CAD and CHIKAKU-MESH developed at the RIKEN and the Japan Atomic Energy Agency (JAEA). The finite element calculations have been carried out with GeoFEM, a parallelized finite element code developed at the Research Organization for Information Science and Technology (RIST), on the Earth Simulator at the Earth Simulator Center of JAMSTEC. Some of figures included in this paper were generated with the Generic Mapping Tools (Wessel and Smith, 1998).

\section{References}

Baba, T., K. Hirata, T. Hori, and H. Sakaguchi, Offshore geodetic data conductive to the estimation of the afterslip distribution following the 2003 Tokachi-oki earthquake, Earth Planet. Sci. Lett., 241, 281-292, 2006.

Dambara, T. and Y. Tomoda, Geodesy and Geophysics, 286pp, Kyoritsushuppan, Tokyo, 1969 (in Japanese).

Hagiwara, Y., Effects of the Pacific and Philippine-Sea plates on the gravity field in central Japan, J. Geod. Soc. Japan, 32, 12-22, 1986 (in Japanese with English abstract).

Hashimoto, M., Finite element modeling of deformations of the lithosphere at an arc-arc junction: The Hokkaido corner, Japan, J. Phys. Earth, 32, 373-398, 1984.

Hashimoto, M., Finite element modeling of the three-dimensional tectonic flow and stress field beneath the Kyushu island, Japan, J. Phys. Earth, 33, 191-226, 1985.

Heki, K., S. Miyazaki, and H. Tsuji, Silent fault slip following an interplate thrust earthquake at the Japan Trench, Nature, 386, 595-598, 1997.

Hirata, K., M. Aoyagi, H. Mikada, K. Kawaguchi, Y. Kaiho, R. Iwase, S. Morita, I. Fujisawa, H. Sugioka, K. Mitsuzawa, K. Suyehiro, H. Kinoshita, and N. Fujiwara, Real-time geophysical measurements on the deep seafloor using submarine cable in the southern Kurile subduction zone, IEEE J. Oceanic Eng., 27, 170-181, 2002.

Hyodo, M. and K. Hirahara, GeoFEM kinematic earthquake cycle simulation in southwest Japan, Pure Appl. Geophys., 161, 2069-2090, 2004.

Iizuka, M., D. Sekita, H. Suito, M. Hyodo, K. Hirahara, D. Place, P. Mora, O. Hazama, and H. Okuda, Parallel simulation system for earthquake generation: fault analysis modules and parallel coupling analysis, Concurrency Computat.: Pract. Exper., 14, 499-519, 2002.

Kanai, T., A. Makinouchi, and A. Nakagawa, Tectonic CAD system and the construction of 3D solid models of tectonic structures, 1999 Japan Earth and Planet. Sci. Joint Meeting, Dg-021, 1999 (in Japanese).

Kanai, T., A. Makinouchi, and Y. Oishi, Development of tectonic CAD/database systems, Int. Workshop on Solid Earth Simulation and ACES WG Meeting, 2000.

Kanai, T., Y. Oishi, A. Makinouchi, T. Homma, and T. Miyamura, CHIKAKU modeling system-Tectonic database/CAD software for predictions of earthquake generation and wave propagation-, Seismol. Soc. Japan 2005 Fall Meeting, P099, 2001 (in Japanese). 
Katsumata, K., N. Wada, and M. Kasahara, Newly imaged shape of the deep seismic zone within the subducting Pacific plate beneath the Hokkaido corner, Japan-Kurile arc-arc junction, J. Geophys. Res., 108, 2565, doi:10.1029/2002JB002175, 2003.

Kawachi, T. and K. Miyashita, 3-dimensional finite element modeling of the subduction and collision of the northernmost part of the Philippine Sea plate, Zisin (J. Seismol. Soc. Japan), 50, 229-240, 1997 (in Japanese with English abstract).

Liu, H.-P., The structure of the Kurile trench-Hokkaido rise system computed by an elastic time-dependent plastic plate model incorporating rock deformation data, J. Geophys. Res., 85, 901-912, 1980.

Masterlark, T., Finite element model predictions of static deformation from dislocation sources in a subduction zone: Sensitivities to homogeneous, isotropic, Poisson-solid, and half-space assumptions, J. Geophys. Res., 108(B11), 2540, doi:10.1029/2002JB002296, 2003.

Masterlark, T., C. DeMets, and H. F. Wang, Homogeneous vs heterogeneous subduction zone models: Coseismic and postseismic deformation, Geophys. Res. Lett., 28, 4047-4050, 2001.

Miura, S., Y. Suwa, A. Hasegawa, and T. Nishimura, The 2003 M8.0 Tokachi-Oki earthquake-How much has the great event paid back slip debts?, Geophys. Res. Lett., 31, doi:10.1029/2003GL019021, 2004.

Miyamura, T., T. Kanai, Y. Oishi, K. Hirahara, T. Hori, M. Hyodo, A. Higashida, T. Hirayama, N. Kato, and A. Makinouchi, Mesh generation of crust structures of southwest Japan by using CHIKAKU system, Proc. Computat. Eng. Conf. of Japan Soc. Computat. Eng. Sci., 9, 521524, 2004 (in Japanese with English abstract).

Miyazaki, S., T. Sato, M. Sasaki, Y. Hatanaka, and Y. Iimura, Expansion of GSI's GPS array, Bull. Geogr. Surv. Inst., 43, 23-36, 1997.

Miyazaki, S., K. M. Larson, K. Choi, K. Hikima, K. Koketsu, P. Bodin, J. Haase, G. Emore, and A. Yamagiwa, Modeling the rupture process of the 2003 September 25 Tokachi-Oki (Hokkaido) earthquake using 1-Hz GPS data, Geophys. Res. Lett., 31, doi:10.1029/2004GL021457, 2004a.

Miyazaki, S., P. Segall, J. Fukuda, and T. Kato, Space time distribution of afterslip following the 2003 Tokachi-oki earthquake: Implications for variations in fault zone frictional properties, Geophys. Res. Lett., 31, doi:10.1029/2003GL019410, 2004b.

Nakajima, J., T. Matsuzawa, A. Hasegawa, and D. Zhao, Seismic imaging of arc magma and fluids under the central part of northeast Japan, Tectonophysics, 341, 1-17, 2001.

Oishi, Y., T. Miyamura, T. Kanai, K. Hirahara, T. Hori, and M. Hyodo, Hexahedral mesh generation of crust structure of south west Japan, 2004 Japan Earth and Planet. Sci. Joint Meeting, S044-P008, 2004 (in Japanese).

Ozawa, S., M. Kaidzu, M. Murakami, T. Imakiire, and Y. Hatanaka, Coseismic and postseismic crustal deformation after the Mw 8 Tokachi-oki earthquake in Japan, Earth Planets Space, 56, 675-680, 2004.
Sato, K., Stress and displacement fields in the northeastern Japan island arc as evaluated with three-dimensional finite element method and their tectonic interpretations, Sci. Rep. Tohoku Univ., Ser. 5, 31, 57-99, 1988.

Sato, K., Numerical experiments on strain migration, J. Geod. Soc. Japan, 35, 27-36, 1989 (in Japanese with English abstract).

Sato, K., H. Ishii, and A. Takagi, Characteristics of crustal stress and crustal movements in the northeastern Japan arc I: Based on the computation considering the crustal structure, Zisin (J. Seismol. Soc. Japan), 34, 551-563, 1981 (in Japanese with English abstract).

Sato, K., S. C. Bhatia, and H. K. Guputa, Three-dimensional numerical modeling of deformation and stress in the Himalaya and Tibetan plateau with a simple geometry, J. Phys. Earth, 44, 227-254, 1996.

Suito, H. and K. Hirahara, Simulation of post-seismic deformations caused by the 1896 Riku-u earthquake, northeast Japan: Re-evaluation of the viscosity in the upper mantle, Geophys. Res. Lett., 26, 2561-2564, 1999.

Wald, D. J. and R. W. Graves, Resolution analysis of finite fault source inversion using one- and three-dimensional Green's functions 2. Combining seismic and geodetic data, J. Geophys. Res., 106, 8767-8788, 2001.

Wessel, P. and W. H. F. Smith, New, improved version of generic mapping tools released, EOS Trans., 79, 579, 1998.

Yoshioka, S. and M. Hashimoto, The stress field induced from the occurrence of the 1944 Tonankai and the 1946 Nankaido earthquakes, and their relation to impending earthquakes, Phys. Earth Planet. Inter., 56, 349-370, 1989a.

Yoshioka, S. and M. Hashimoto, A quantitative interpretation on possible correlations between intraplate seismic activity and interplate great earthquakes along the Nankai trough, Phys. Earth Planet. Inter., 58, 173-191, 1989b.

Yoshioka, S. and H. Suzuki, Effects of three-dimensional inhomogeneous viscoelastic structures on quasi-static strain and stress fields associated with dislocation on a rectangular fault, Zisin (J. Seismol. Soc. Japan), 50, 277-289, 1997 (in Japanese with English abstract).

Yoshioka, S., M. Hashimoto, and K. Hirahara, Displacement fields due to the 1946 Nankaido earthquake in a laterally inhomogeneous structure with the subducting Philippine sea plate-a three-dimensional finite element approach, Tectonophysics, 159, 121-136, 1989.

Zhao, D., S. Horiuchi, and A. Hasegawa, Seismic velocity structure of the crust beneath the Japan islands, Tectonophysics, 212, 289-301, 1992.

Zhao, D., A. Hasegawa, and H. Kanamori, Deep structure of Japan subduction zone as derived from local, regional and teleseismic events, $J$. Geophys. Res., 99, 22313-22329, 1994.

K. Sato (e-mail: sato@u-gakugei.ac.jp), N. Minagawa, M. Hyodo, T. Baba, T. Hori, and Y. Kaneda 